\title{
Electrochemical dehalogenation of disinfection by-products and iodine-containing contrast media: A review
}

\author{
Gregory Korshin ${ }^{1+}$, Mingquan Yan $^{2}$ \\ ${ }^{1}$ Department of Civil and Environmental Engineering, University of Washington, Box 352700, Seattle, WA 98195-2700, USA \\ ${ }^{2}$ Department of Environmental Engineering, Peking University, the Key Laboratory of Water and Sediment Sciences, Ministry of Education, Beijing \\ 100871, China
}

\begin{abstract}
This paper summarizes results of research on the electrochemical (EC) degradation of disinfection by-products (DBPs) and iodine-containing contrast media (ICMs), with the focus on EC reductive dehalogenation. The efficiency of EC dehalogenation of DBPs increases with the number of halogen atoms in an individual DBP species. EC reductive cleavage of bromine from parent DBPs is faster than that of chlorine. EC data and quantum chemical modeling indicate that the EC reduction of iodine-containing DBPs (I-DBPs) is characterized by the formation of active iodine that reacts with the organic substrate. The occurrence of ICMs has attracted attention due to their association with the generation of I-DBPs. Indirect EC oxidation of ICMs using anodes that produce reactive oxygen species can result in a complete degradation of these compounds yet I-DBPs are formed in the process. Reductive EC deiodination of ICMs is rapid and its overall rate is diffusion-controlled yet I-DBPs are also produced in this reaction. Further progress in practically feasible EC methods to remove DBPs, ICMs and other trace-level organic contaminants requires the development of novel electrocatalytic materials, elimination of mass transfer limitations via innovative design of $3 \mathrm{D}$ electrodes and EC reactors, and further progress in the understanding of intrinsic mechanisms of EC reactions of DBPs and TrOC at EC interfaces.
\end{abstract}

Keywords: Disinfection by-products, Electrochemistry, Iodine-containing media, Reductive dehalogenation, Trace organic contaminants, Water treatment

\section{Introduction}

The occurrence and effects of disinfection by-products (DBPs) and trace-level organic contaminants (TrOCs) have attracted growing attention of the environmental community $[1,2]$. In the case of DBP controls, current regulations tend to focus on two most prominent, on the mass basis, DBP groups of trihalomethanes (THMs) and haloacetic acids (HAAs) [3, 4]. However, ongoing research shows that actual health effects may be associated with DBPs other than HAAs and THMs [5, 6]. Such species include, among others, N-DBPs (e.g., haloacetonitriles and haloacetamides) and I-DBPs (e.g., monoiodoacetic acid (MIAA)) [7, 8]. Some TrOCs groups, notably iodine-containing contrast media (ICMs) used in medical imaging [9, 10] and frequently encountered in the environment $[11,12]$ have been shown to be precursors of I-DBPs formed via the degradation of ICMs by halo- gen-based disinfectants or reactive oxygen species (ROS) formed in advanced oxidation processes (AOPs), as well as in EC treatment that involves both oxidation and reduction [13-16].

Adverse effects associated with DBPs and TrOCs and the difficulties in preventing their generation and/or removal by conventional techniques necessitate the development of alternative treatment methods. Electrochemical (EC) methods constitute a distinct group of such methods because they employ reactions induced by electric current passing across the interface between an EC-controlled working electrode, or a system of electrodes, and the solution being treated. This process can be accompanied by direct interactions of a target compound with the electrons being released or consumed at the interface. In this case the EC treatment can be termed direct. Alternatively, an EC process may result in the formation of oxidants (e.g., ROS, halogen species) or reductants that react with the target contaminant in
This is an Open Access article distributed under the terms of the Creative Commons Attribution Non-Commercial License (http://creativecommons.org/licenses/by-nc/3.0/) which permits unrestricted non-commercial use, distribution, and reproduction in any medium, provided the original work is properly cited.

Copyright (C) 2018 Korean Society of Environmental Engineers
Received January 30, 2018 Accepted March 20, 2018

${ }^{\dagger}$ Corresponding author

Email: korshin@uw.edu

Tel: +1-206-543-2394

ORCID: 0000-0002-9226-6601 
solution bulk but not necessarily at the electrode/solution interface $[17,18]$. Such mode of EC treatment can be termed indirect.

EC control of water treatment processes offers important benefits, for instance i) ease of on-demand operations and the possibility of a complete automation of water treatment, ii) little or no need to use externally stored chemicals, iii) options to control and adjust the EC potential of the working electrode(s) to optimize the degradation of target compounds and suppress background reactions (e.g., reduction or oxidation of water), and iv) a wide range of EC reactor configurations and available electrode materials, including those with electrocatalytic properties.

These aspects of EC processes as well pertinent theoretical and practical challenges have been examined in a number of prior reviews and related publications, for instance [18-20]. Given the vast amount of data generated in the studies of EC-driven processes relevant to water treatment, this review will henceforth focus on two specific EC applications, namely on the EC-controlled reductive dehalogenation of DBPs and EC degradation of ICMs.

\section{EC-controlled Direct Reductive Dehalogenation of DBPs}

\subsection{Reductive EC Dehalogenation of Haloacetic Acids}

Reductive EC dehalogenation (that is, the EC reactions that cause the cleavage of halogen atoms from the parent molecule) of HAAs which constitute an important class of DBPs has been addressed in considerable detail over the last two decades [21]. In a relatively early study, Korshin and Jensen [22] showed that di- and trihalogenated chlorine- and bromine-containing HAAs exhibit notable EC activity at the surface of cathodically polarized gold and copper rotating disk electrodes (RDE). The current associated with the EC reduction of HAAs was considerably higher in the case of copper. The EC reduction currents increased with the number of halogen atoms in HAA molecules. All HAAs except monochloroacetic acid (MCAA) were found to be susceptible to EC dehalogenation which was especially rapid from Br-containing HAAs. The formation of MCAA with its low EC activity yet a potentially considerable toxicity was judged to be a limiting factor in the developed of EC methods to removes HAAs.

These trends were in agreement with those observed in Hozalski et al. [23] for the case of the reduction of trihalogenated HAAs by zero-valent iron (ZVI), which is widely present in drinking water distribution networks and other engineered systems. (While the reduction of HAAs or other DBPs/TrOC by ZVI is not explicitly EC-controlled, it involves the coupling of the EC rate of Fe oxidation and that of the reduction of the involved contaminants or dissolved oxygen. As such, these processes can be considered to constitute a subtype of EC reactions). Experiments with trichloro-, tribromo-, dibromochloro- and bromodichloroacetic acids (TCAA, TBAA, DBCAA and BDCAA, respectively) showed that these compounds were rapidly dechlorinated by ZVI. The bromine atoms in HAAs were removed faster than chlorine in these reactions. TBAA was observed to undergo a complete dehalogenation to form acetate while the reduction of the other examined HAAs tended to stop at the formation of MCAA. The rate of ZVI-induced dehalogenation was highest for DBCAA, in which case it exceeded the rate of BDCAA or TBAA dehalogenation by an order of magnitude. The dehalogenation of TCAA was more than two orders of magnitude slower than that of DBCAA. Comparison of the relative effects of electron and mass transfer limitations on the EC dehalogenation of HAAs by ZVI [24] showed that the dehalogenation of TBAA, DBCAA and BDCAA was limited by mass transfer. Mass transfer had a limited effect on the reduction of DBAA and BCAA while the reduction of TCAA, DCAA, BCAA and MCAA was limited by the rate of electron transfer from the cathode to the involved HAA species. The presence and concurrent EC reduction of dissolved oxygen had relatively little effect on these processes.

Higher rates of the direct EC debromination of trihalogenated HAAs were also observed in the recent study concerned with the development of EC sensors for DBPs [25]. Voltammetric EC data presented in that study indicate, in accord with the results of Hozalski et al. [23], that the EC reductive cleavage of $\mathrm{Br}$ from TBAA, BDCAA and DBCAA occurs in the range of potentials 0 to $-0.5 \mathrm{~V}$ while the cleavage of $\mathrm{Cl}$ from these DBPs tends to become prominent in the range of potentials -0.5 to $-1 \mathrm{~V}$. Differences in the voltammetric data generated using a gold electrode allowed quantitating the examined HAA species at $\mu \mathrm{g} / \mathrm{L}$ levels. In another study focused on analytical aspects of HAA quantitation [26], the reduction of MCAA, DCAA and TCAA on a catalytically active poly-Ni(II)-tetrasulfonated phthalocyanine (poly-NiTSPC) film formed on the gold substrate was compared with that on bare Au surface. The presence of the poly-NiTSPC film was observed to decrease the EC potential of the onset of the reduction of the examined HAAs by ca. $0.25 \mathrm{~V}$.

\subsection{Effects of EC Conditions and Electrode Materials on the EC Reduction of HAAs}

The results discussed above show that the EC reduction of HAAs and other DBPs can be accelerated using electrocatalytic materials. One type of a potentially promising electrode material suitable for DBP treatment is graphene whose performance as a component of a composite 3D graphene-Cu foam electrode was examined in Mao et al. [27]. This material was used for EC reductive dehalogenation of TCAA. Voltammetric and EC impedance spectra generated for this system showed that the presence of graphene caused the reduction of TCAA to accelerate. Mechanistically, this effects was tied to the involvement of atomic $\mathrm{H}^{*}$ adsorbates. The EC reduction of TCAA using this material resulted in the formation of dichloroacetic acid (DCAA) and MCAA although levels of acetic acid comparable with those of MCAA were also observed. This indicates that the use of electrocatalytic materials may allow achieving a complete dehalogenation of monohalogenated aliphatic DBPs that typically show little EC activity when conventional electrode materials are used.

Effects of electrocatalysis have been frequently examined for 
inexpensive materials (graphite, iron) modified with $\mathrm{Pd}$, Pt or other catalysts. Li et al. [28] used a Pd/Fe-modified carbon electrode to carry out EC reduction of MAA, DCAA and TCAA at $-1.5 \mathrm{~V}$ vs. standard calomel electrode (SCE) and $\mathrm{pH}$ 3. The deposition of $\mathrm{Pd} / \mathrm{Fe}$ resulted in the acceleration of the degradation of these HAAs, compared with their removal using the unmodified carbon electrode. As was observed in other studies, the reductive dechlorination of TCAA and DCAA was relatively rapid but the reduction of MCAA to acetic acid was quite slow and deemed to proceed via the indirect reduction of MCAA by atomic $\mathrm{H}^{*}$ generated at $\mathrm{Pd}$ nuclei formed on the electrode surface. The acceleration of the EC reduction of HAAs by atomic $\mathrm{H}^{*}$ was also observed in another study [29] which utilized an EC-controlled bimetallic Pd/In catalyst that promoted the formation of atomic $\mathrm{H}^{*}$. The catalyst was incorporated into an EC reactor whose operations resulted in a rapid removal of TCAA yet MCAA again was observed to form as a final product.

The removal of MCAA formed in EC and related treatment processes (e.g., ZVI) may be addressed by combining the EC dehalogenation of the target with a post-treatment process. The improved performance of a combined approach was demonstrated for the degradation of TCAA by ZVI followed by the treatment of the ZVI effluent containing DCAA and MCAA by biologically active carbon [30]. This process was shown to result in a considerable reduction of MCAA concentrations.

The removal of HAAs using Pd-modified granular activated carbon (GAC) and EC-controlled Pd-modified carbon paper cathode was examined in Zhao et al. [31]. Moderate levels of the removal of HAAs at relatively low current densities $\left(0.3 \mathrm{~mA} / \mathrm{cm}^{2}\right)$ were observed. For that current density, the rate of HAA removal was judged to be affected by both electron transfer and diffusion rates. For higher current densities (e.g., $0.6 \mathrm{~mA} / \mathrm{cm}^{2}$ ), diffusion controls of the reaction rate became more prominent.

\subsection{EC Degradation of DBPs Other Than HAAs}

The EC-driven dehalogenation of DBPs other than HAAs has been addressed in a number of studies. Li et al. [32] reported data for a process that combined the sorption of DBPs by GAC and EC-driven electrolysis applied to dehalogenate the adsorbed DBPs. GAC adsorption effectively removed a representative range of DBPs (a mixture of haloacetonitriles, trihalomethanes, haloacetaldehydes, haloacetamides and chloropicrin was tested). Further treatment by electrolysis carried using the GAC cathode polarized at $-1.0 \mathrm{~V}$ vs. standard hydrogen electrode (SHE) resulted in the degradation of the retained DBPs whose levels decreased by up to $90 \%$ for a relatively long treatment times (6 to 12 h). Activated carbons and charcoal were more effective than graphite for electrolysis, primarily due to a much higher sorption capacity of the former class of materials. The reduction of the examined DBPs was concluded to proceed via direct EC dehalogenation that results in the formation of $\mathrm{Cl}^{-}, \mathrm{Br}^{-}$and $\mathrm{I}^{-}$ions. The involvement in the reduction of the retained DBPs of redox-active functional groups typical for activated carbons, for instance phenols and quinones, was also hypothesized. Similar trends were reported for the case of EC dehalogenation of DBPs using a resin-impregnated graphite cathode [19]. However, the relatively low degradation efficiencies (in a $20 \%$ - 95\% removal range, with most species degraded at ca. $60 \%$ ) observed for $>24 \mathrm{~h}$ treatment duration suggested that this technology may be best applied for specific types of DBP-containing effluents.

ZVI-driven degradation of halonitromethanes (HNM) exemplified by mono-, di- and trichloronitromethans (MCNM, DCNM and TCNM, respectively) was examined in [33]. In the presence of a high ZVI dose (2 to $4 \mathrm{~g} / \mathrm{L}$ of ZVI) and $\mathrm{pH} 7.5$, the examined HNM species underwent a relatively slow (within one to four hours) degradation. The apparent rate of dechlorination decreased with the number of chlorine atoms in HNM molecules while the nominal end product of their dechlorination, nitromethane was observed to undergo rapid conversion by ZVI to methylamine. The degradation of TCNM and DCNM was concluded to proceed via parallel pathways of hydrogenolysis and $\alpha$-elimination. These reactions take place in drinking water distribution systems containing iron material (e.g., cast or ductile iron pipe).

\subsection{Specific Aspects of the Deiodination of I-DBPs}

Specific aspects of the EC reduction of I-DBPs and mechanisms of deiodination were reported in Ma et al. [34]. That study examined cathodic reactions of iodoform and MIAA at varying mass transfer conditions controlled by electrode rotation and determined that these I-DBPs undergo facile deiodination at EC potentials $<-0.3 \mathrm{~V}$ vs. standard $\mathrm{Ag} / \mathrm{AgCl}$ electrode (SSCE). Mechanisms of the EC deiodination of MIAA and iodoform were shown to be similar and in both case the cleavage of iodine atoms from the parent molecule was accompanied by the formation of active iodine intermediates. This result was unexpected as the classical mechanism of EC dehalogenation favors the cleavage of $\mathrm{X}^{-}$ions in the first of the two electron transfer steps typical for reductive dehalogenation of chlorine- and bromine-containing compounds [35, 36]. Quantum chemical modeling of the electron transfer reactions involved in the reductive dehalogenation of trihalomethanes confirmed that in the case of $\mathrm{C}-\mathrm{Cl}$ and $\mathrm{C}-\mathrm{Br}$ bonds the formation of $\mathrm{Cl}^{-}$and $\mathrm{Br}^{-}$ions is thermodynamically preferable for the first electron transfer while the reductive cleavage of the C-I bond is likely to proceed via the formation of I intermediate which then forms active iodine species.

The above analysis is not meant to be exhaustive but it is sufficient to demonstrate that DBP removal methods based on their EC-controlled reductive dehalogenation are fundamentally feasible, especially in the case of hybrid methods that combine EC-driven and conventional processes, for instance GAC adsorption and/or biodegradation. The EC reductive dehalogenation of DBPs has been well studied for HAAs and in some extent THMs yet for many other individual DBP species and their general classes (e.g., N- and I-DBPs, polar phenolic DBPs, etc.) more research is needed to quantify the kinetics of their EC reactions and effects of electrode properties on them. The nature of intermediates and final products formed via the EC reduction of halogenated DBPs also needs to be ascertained in more detail. Finally, practical implementation of treatment processes based on the EC reductive dehalogenation of DBPs clearly depends 
on further development of new types of electrocatalytic materials amenable for incorporation into EC reactors that can overcome mass transfer limitations typical for EC treatment of trace-level contaminants.

\section{Electrochemical Degradation of lodinated Contrast Media}

ICMs are widely encountered in hospital wastewaters, municipal wastewater and drinking water at concentrations up to $100 \mu \mathrm{g} / \mathrm{L}$ [12-16, 37-39]. (Chemical structures of those ICMs that have been used in prior studies of EC treatment are shown in Fig. 1). Conventional processes have little success in removing ICMs due to their high hydrophilicity and resistance to biological degradation. AOPs such as UV photolysis, sonolysis, Fenton, photo-Fenton, $\mathrm{H}_{2} \mathrm{O}_{2} / \mathrm{UV}-\mathrm{C}$, $\mathrm{TiO}_{2} / \mathrm{UV}-\mathrm{A}$ and $\mathrm{O}_{3} / \mathrm{H}_{2} \mathrm{O}_{2}$ remove ICMs but these processes generate toxic iodinated by-products [14-16, 37-39].

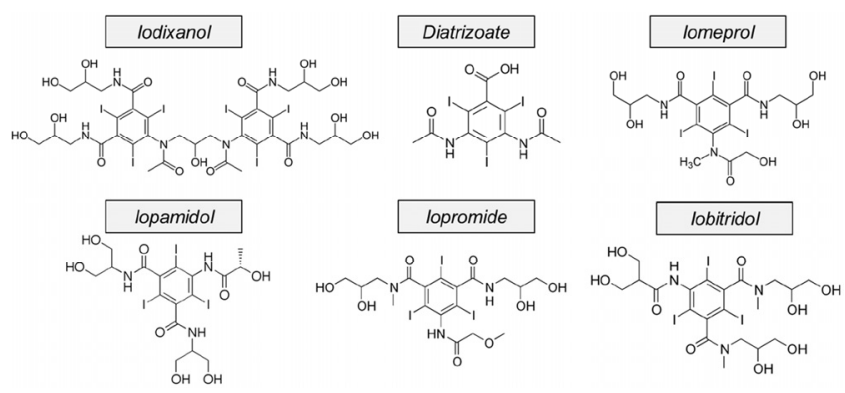

Fig. 1. Structures of ICMs used in the reported studies of their electrochemical behavior.

\subsection{Reductive Deiodination of ICMs}

Reductive dehalogenation (referred to in the case of ICMs as deiodination) induced via external EC polarization of a working electrode or promoted by zero-valent metals and metal-based catalysts has shown promise in the treatment of ICMs [13, 40-42]. The susceptibility of ICMs to reductive degradation was demonstrated by Zwiener et al. [12] who examined the EC reduction of iomeprol on nickel cathode. Sequential cleavage of up to three iodine atoms from the parent molecule was observed to occur and mono- and di-deiodinated intermediates with a relatively low stability were formed in this process. The end product of the EC reduction of iomeprol was suggested to form via the transformation of the completely deiodinated iomeprol and a loss of a $(\mathrm{C}=\mathrm{O}) \mathrm{CHOH}$ group from the side chain. The authors concluded that EC degradation of ICMs may be a feasible option for the removal of these compounds from urine of patients or hospital effluents.

\subsection{Reduction of ICMs by ZVI}

The removal of ICMs and several antineoplastic agents by ZVI in the presence of oxygen was demonstrated in $\mathrm{Mu}$ et al. [43]. That study showed the removal of these species followed a pseu- do-first order kinetics. The removal of iopromide was relatively slow requiring $>6 \mathrm{~h}$ of contact time at $\mathrm{pH} 3$ to remove $>$ $90 \%$ of the initially present iopamidol. The reaction rates were strongly affected by $\mathrm{pH}$ and hydrodynamic conditions which indicated the presence of mass transfer limitations.

The ZVI-driven reduction of iopamidol in the presence of monochloramine was studied in Dong et al. [41]. The contact times were as long as $60 \mathrm{~h}$ although the removal of iopamidol was most notable within the initial $10 \mathrm{~h}$ of reaction time. The degradation of iopamidol was more rapid when the corrosion of ZVI was promoted by sulfate or chloride ions at low $\mathrm{pH}$ (e.g., $\mathrm{pH}$ 5) and it became slower as the surface became passivated. The deiodination of iopamidol by increasing amounts of ZVI was accompanied by the increases of the formation of I-DBPs such as $\mathrm{CHCl}_{2}, \mathrm{CHI}_{3}$, MIAA, diodo- and triiodoacetic acids (DIAA and TIAA, respectively) albeit small amounts of monoiododichloromethane and MIAA were formed in the absence of zero-valence iron. Molar yields of iodoform and TIAA formed in the case of ZVI-induced degradation of iopamidol at $\mathrm{pH} 5$ were ca. $1 \%$. They decreased monotonically with $\mathrm{pH}$ and were ca. $<0.1 \%$ at $\mathrm{pH}$ 9. The authors expressed an opinion that given the ubiquity of ZVI in drinking water systems that about with unlined cast iron distribution pipes, the EC-controlled deiodination of iopamidol that may be present in source surface water may result in increased levels of I-DBPs.

$\mathrm{Hu}$ et al. [42] found that typical corrosion products found on the surface of pipes in drinking water systems (e.g., $\mathrm{CuO}$ and $\delta-\mathrm{MnO}_{2}$ ) may affect the activity of peroxymonosulfate in its reactions with iopamidol. The rate of iopamidol degradation by persulfate was observed to increase in the presence of $\mathrm{CuO}$ or $\delta-\mathrm{MnO}_{2}$, especially in the case of the former solid which was hypothesized to promote the formation of sulfate radical while $\mathrm{CuO}$ promoted the generation of $\mathrm{OH}$ radicals. The majority of the iodine released from iopamidol upon its oxidation by persulfate was oxidized to iodate yet ca. $5 \%$ of the iodine initially present in the system was transformed to active iodine species.

The degradation of iopamidol at $\mathrm{pH} 3$ in the presence zero valent aluminum (ZVA) (a $1 \mathrm{~g} / \mathrm{L} \mathrm{ZVA}$ dose) was also observed to be slow. In the absence of hydrogen peroxide or persulfate only ca. $40 \%$ of the initially present iopamidol was removed within ca. 2 hours [9]. The combination of ZVA and persulftate accelerated the process in distilled water but in surface water the extent of iopamidol removal was lower, and little iopamidol was removed when wastewater was treated using ZVA/persulfate.

\subsection{Effects of EC Conditions and Electrode Materials on the Degradation of ICMs}

Examination of the EC degradation of $100 \mu \mathrm{M}$ iopromide in a batch reactor equipped with a boron doped diamond (BDD) anode and a platinum cathode showed that $>90 \%$ on the initial compound could be mineralized for treatment time $>7.5 \mathrm{~h}$ [44]. Shorter treatment times resulted in the generation of oxidatively and reductively formed byproducts and deiodinated iopromide which was toxicologically inert. Further experiments utilizing a divided cell setup showed that the pathway of the degradation of iopromide in the examined system involved parallel re- 
actions of reductive EC deiodination at the cathode, direct oxidation at the BDD surface and indirection radical oxidation and hydrolysis. The EC treatment of iopromide resulted in the preferential formation of iodide compared with that of iodate $(90 \%$ and $10 \%$ of the total, respectively).

EC reduction of the ICM diatrizoate using three-dimensional graphite felt and graphite felt doped with nanoparticles was examined in Radjenovic et al. [13]. The presence of palladium nanoparticles resulted in a significant enhancement of the removal of diatrizoate and enabled its complete deiodination to 3,5-diacetamidobenzoic acid. When the system was employed in the treatment of hospital wastewater, diatrizoate was reduced, but the extent of EC reduction decreased as a result of competing reactions. Following the EC reduction of diatrizoate to 3,5-diacetamidobenzoic acid, the next step of EC oxidation with BDD anodes was employed to remove 3,5-diacetamidobenzoic acid. The EC reactions were employed to degrade diatrizoate in a three-compartment EC reactor operated in a continuous mode. This allowed achieving a complete deiodination of diatrizoate at a $-1.7 \mathrm{~V}$ vs. SHE cathode potential while a predominant part (ca. $80 \%$ ) of the released iodide ions was electrodialyzed into a central compartment. The oxidation of the effluent formed from the cathode compartment at the BDD anode resulted in the further removal of the reduction products.

An example of a combined system that incorporated the reductive EC dehalogenation of iopromide and the treatment of the EC effluent with a biologically enhanced anodic oxidation enhanced by acetate feeding was presented in $\mathrm{Mu}$ et al. [43]. The system used a granular graphite cathode whose potential was varied between -0.5 to $-0.9 \mathrm{~V}$ vs SHE. The cathodic shift of the potential of the working electrode resulted in a strong acceleration of the EC removal of iopromide and its complete deiodination at potentials $<-0.8 \mathrm{~V}$ although the Coulombic efficiency of the dehalogenation of iopromide at the cathode was $<1 \%$. The Coulombic efficiency of the acetate oxidation at the graphite anode was estimated to be somewhat $<50 \%$. Several intermediates formed upon the reduction of iopromide were observed.

The EC oxidation of six ICMs (amidotrizoate also known as diatrizoate, iopamidol, iobitridol, iodixanol, iopromide and iomeprol) in batch experiments that employed dimensionally stable anodes (DSA) resulted in the removal of up to $85 \%$ of the examined ICMs for electrolysis time exceeding $2.5 \mathrm{~h}$ at a constant current [45]. However, the polarity of the employed DSA electrodes was switched during the treatment, and both anodic and cathodic reactions took place in the system. The EC treatment was concluded to result in the reductive deiodination of the examined ICMs, further reduction of the resultant alkyl aromatic amides to simple amides and the de-acylation of $\mathrm{N}$-aromatic amides to aromatic amines. The consumption of energy in the examined process was high $\left(>370 \mathrm{kWh} / \mathrm{m}^{3}\right)$ yet the reported process was deemed by the authors to be viable to treat hospital wastewater or other pharmaceutical waste-contaminated streams.

The studies discussed in this section show that while the EC reductive deiodination of ICMs as well as the oxidation of their degradation products is reasonably facile, several challenges are associated with these processes. For instance, the treatment of ICM-containing solutions using zero valence metals even at their high doses tends to be slow, may require using low $\mathrm{pHs}$ and additional oxidants such as hydrogen peroxide or persulfate. On the other hand, the EC reduction of ICMs is facile and tends to be limited by mass transfer to the electrode surface. The EC reduction of ICMs is accompanied by the generation of unstable iodine-containing intermediates and, ultimately, completely deiodinated products such as diacetamidobenzoic acid and aromatic amines. These products have been observed to be amenable to removal by anodic oxidation and/or biological treatment.

\subsection{Mechanisms of the EC Deiodination of ICMs}

A challenging aspect of the EC treatment of ICMs is that this process is accompanied by the release of iodide (and in some extent iodate) cleaved from the parent ICMs via either EC reductive dehalogenation or in oxidative transformations of ICM molecules or intermediates formed upon their degradation. In the case of reductive EC deiodination, formation of active iodine species via the mechanisms similar to those observed for I-DBPs $[34,46]$ can be anticipated. Examination of the EC reduction of iopamidol and diatrizoate by the rotating ring-disk electrode (RRDE) method [46] showed unambiguously that in the range of potentials ca. -0.6 to $-0.9 \mathrm{~V}$ vs. SSCE the EC reduction of these ICMs was mass transfer-controlled. The potentiodynamic data indicated that the EC-induced cleavage of the iodine atoms from the parent ICMs was nearly simultaneous for the three incorporated iodine atoms. The presence of natural organic matter (NOM) and model compounds such as resorcinol, catechol and guaicol did not affect the EC reduction of the ICMs but active iodine species formed as a result of the EC-induced transformations of these ICMs reacted readily with NOM and model compounds.

The formation of active iodine species in the case of the EC reduction of ICMs as well as the oxidation of the released iodide to active iodine by disinfectants can lead to the formation of I-DBPs. The scheme of reactions taking place in the case of electrochemical reduction of ICM compounds was shown in Fig. 2. Thus the control of I-DBPs formed in EC treatment of ICMs becomes a priority. This necessitates the development of reactors in which the released iodide is intercepted and/or removed, for instance by electrodialysis used in [13] or other techniques. As in the case of the EC reductive treatment of

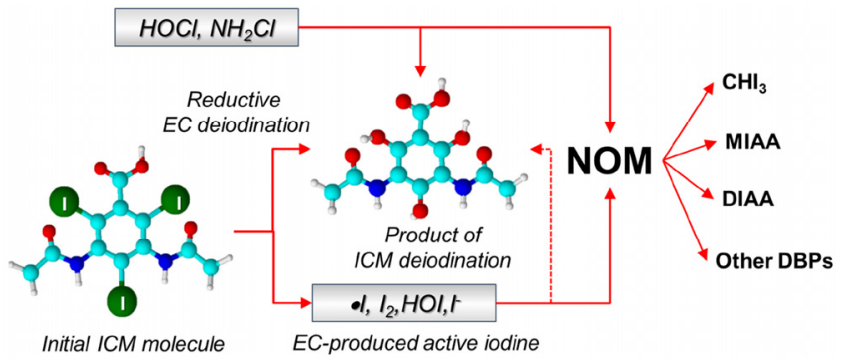

Fig. 2. Suggested scheme of EC deiodination of ICMs and attendant formation of I-DBPs. 


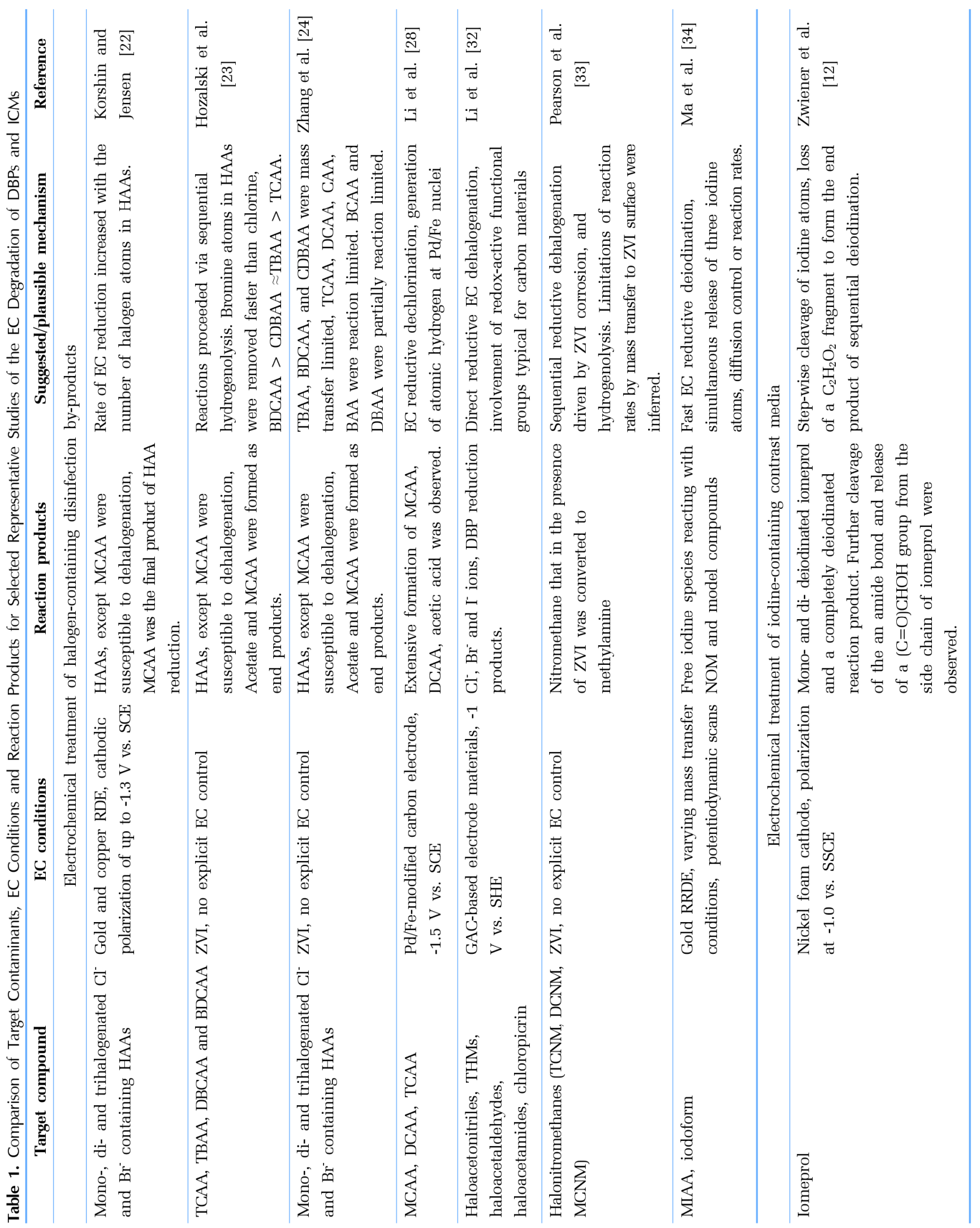




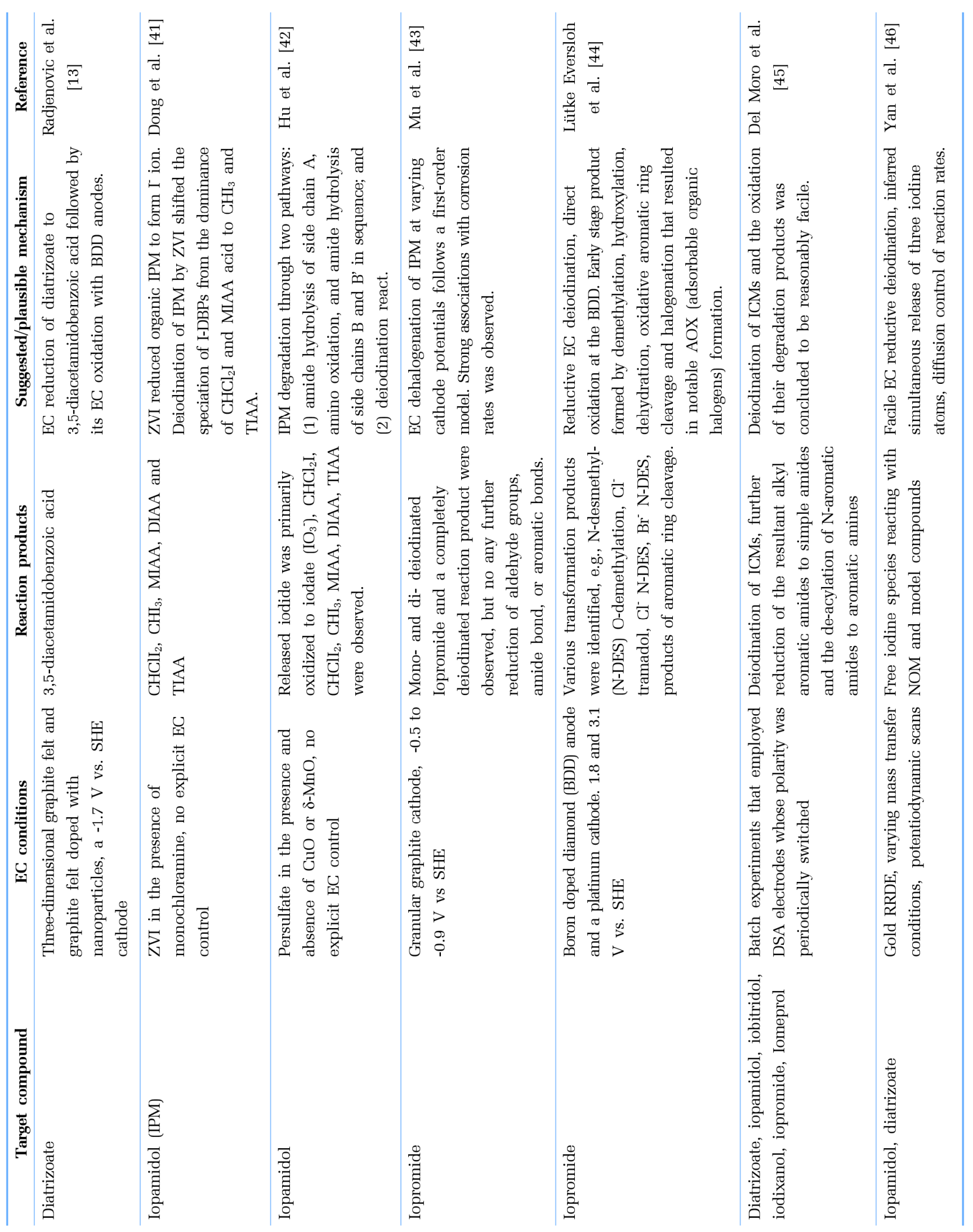


halogenated DBPs, combining EC processes with appropriate pre- and post-treatment options may be highly beneficial for the development of practically feasible EC-based treatment processes for the removal of ICMs and other TrOCs.

\section{Conclusions and Suggestions for Future Research}

This paper summarizes results of research concerned with the EC degradation of halogen-containing DBPs and ICMs. The efficiency of EC dehalogenation of DBPs increases with the number of halogen atoms present in a particular individual DBP species (e.g., mono-, di- and trihaloacetic acids). EC-induced reductive cleavage of bromine from parent DBPs is considerably faster than that of chlorine, and it occurs at lower cathodic potentials. EC data and quantum chemical modeling indicate that the EC reduction of iodine-containing DBPs proceed via a pathway that is different from the classical dehalogenation sequence in which the transfer of the first electron in the two-step sequential electron transfer sequence is accompanied by the cleavage of a halide $\mathrm{X}^{-}$anion. The EC reduction of I-DBPs is characterized by the formation of active iodine that reacts readily with the organic substrate thus potentially leading to the formation of secondary I-DBPs. EC oxidation of ICMs using anodes that produce reactive oxygen species (e.g., BDD, DSA) combined with the cathodic reduction of ICMs can result in a complete degradation these compounds yet iodine-containing DBPs are formed in the process. Reductive EC deiodination of ICMs using various cathode materials (e.g., nickel, platinum, graphite) is rapid and the overall rate of the EC degradation of ICM is diffusion-controlled. Deiodination of ICMs can also occur in drinking water networks via the engagement of iron surfaces and corrosion products typical for these systems. The need to intercept and remove active iodine species and iodide ions formed upon the EC degradation of ICMs is a specific challenge characteristic for the treatment of this class of TrOCs. Further progress in practically feasible EC methods to remove DBPs, ICMs and other TrOCs requires the development of more selective and stable electrocatalytic materials, elimination of mass transfer limitations via innovative design of 3D electrodes and EC reactors, and a more detailed understanding of intrinsic mechanisms of EC reactions of DBPs and TrOC at EC interfaces. Another high priority is the development of hybrid treatment systems that combine EC-controlled reactors with other pre- or post-treatment techniques.

\section{Acknowledgments}

This study was partially supported by the National Science Foundation of China (projects No. 51578007 and No. 51721006). Gregory Korshin thanks the Foreign Experts Program of China for supporting his work at Peking University. The views represented in this publication do not necessarily represent those of the funding agencies.

\section{References}

1. Richardson SD, Thruston AD, Caughran TV, Chen PH, Collette TW, Floyd TL. Identification of new drinking water disinfection byproducts formed in the presence of bromide. Environ. Sci. Technol. 1999;33:3378-3383.

2. Barbosa MO, Moreira NFF, Ribeiro AR, Pereira MFR, Silva AMT. Occurrence and removal of organic micropollutants An overview of the watch list of EU Decision 2015/495. Water Res. 2016;94:257-279.

3. Richardson S, Postigo C. Drinking water disinfection by-products. Emerging organic contaminants and human health. Barceló D, ed. Springer Berlin Heidelberg; 2012. p. 93-137.

4. Allard S, Tan J, Joll CA, von Gunten U. A mechanistic study on the formation of $\mathrm{Cl}-/ \mathrm{Br}-/ \mathrm{I}-$ Trihalomethanes during chlorination/chloramination combined with a theoretical cytotoxicity evaluation. Environ. Sci. Technol. 2015;49:11105-11114

5. Zhai H, Zhang X. Formation and decomposition of new and unknown polar brominated disinfection byproducts during chlorination. Environ. Sci. Technol. 2011;45:2194-2201.

6. Zhai H, Zhang X, Zhu X, Liu J, Ji M. Formation of brominated disinfection byproducts during chloramination of drinking water: New polar species and overall kinetics. Environ. Sci. Technol. 2014;48:2579-2588.

7. Plewa MJ, Wagner ED, Richardson SD, Thruston AD, Woo Y-T, McKague AB. Chemical and biological characterization of newly discovered iodoacid drinking water disinfection byproducts. Environ. Sci. Technol. 2004;38:4713-4722.

8. Richardson SD, Fasano F, Ellington JJ, et al. Occurrence and mammalian cell toxicity of iodinated disinfection byproducts in drinking water. Environ. Sci. Technol. 2008;42:8330-8338.

9. Arslan-Alaton I, Olmez-Hanci T, Korkmaz G, Sahin C Removal of iopamidol, an iodinated X-ray contrast medium, by zero-valent aluminum-activated $\mathrm{H}_{2} \mathrm{O}_{2}$ and $\mathrm{S}_{2} \mathrm{O}_{8}{ }^{2-}$. Chem. Eng. J. 2017;318:64-75.

10. Kormos JL, Schulz M, Ternes TA. Occurrence of iodinated $\mathrm{X}$-ray contrast media and their biotransformation products in the urban water cycle. Environ. Sci. Technol. 2011;45: 8723-8732.

11. Weissbrodt D, Kovalova L, Ort C, et al. Mass flows of X-ray contrast media and cytostatics in hospital wastewater. Environ. Sci. Technol. 2009;43:4810-4817.

12. Zwiener C, Glauner T, Sturm J, Woerner M, Frimmel FH. Electrochemical reduction of the iodinated contrast medium iomeprol: Iodine mass balance and identification of transformation products. Anal. Bioanal. Chem. 2009;395:1885-1892.

13. Radjenovic J, Flexer V, Donose BC, Sedlak DL, Keller J. Removal of the X-ray contrast media diatrizoate by electrochemical reduction and oxidation. Environ. Sci. Technol 2013;47:13686-13694.

14. Duirk SE, Lindell C, Cornelison CC, et al. Formation of toxic iodinated disinfection by-products from compounds used in medical imaging. Environ. Sci. Technol. 2011;45:6845-6854.

15. Tian FX, Xu B, Lin YL, Hu CY, Zhang TY, Gao NY. Photodegradation kinetics of iopamidol by UV irradiation and enhanced formation of iodinated disinfection by-prod- 
ucts in sequential oxidation processes. Water Res. 2014;58: 198-208.

16. Ye T, Xu B, Wang Z, et al. Comparison of iodinated trihalomethanes formation during aqueous chlor(am)ination of different iodinated X-ray contrast media compounds in the presence of natural organic matter. Water Res. 2014;66:390-398.

17. Bergmann MEH, Rollin J. Product and by-product formation in laboratory studies on disinfection electrolysis of water using boron-doped diamond anodes. Catal. Today 2007;124: 198-203.

18. Särkkä H, Bhatnagar A, Sillanpää M. Recent developments of electro-oxidation in water treatment - A review. $J$. Electroanal. Chem. 2015;754:46-56.

19. Radjenovic J, Farre MJ, Mu Y, Gernjak W, Keller J. Reductive electrochemical remediation of emerging and regulated disinfection byproducts. Water Res. 2012;46:1705-1714.

20. Radjenovic J, Sedlak DL. Challenges and opportunities for electrochemical processes as next-generation technologies for the treatment of contaminated water. Environ. Sci. Technol. 2015;49:11292-11302.

21. Peters DG, McGuire CM, Pasciak EM, et al. Electrochemical dehalogenation of organic pollutants. J. Mex. Chem. Soc. 2014;58:287-302.

22. Korshin GV, Jensen MD. Electrochemical reduction of haloacetic acids and exploration of their removal by electrochemical treatment. Electrochim. Acta 2001;47:747-751.

23. Hozalski RM, Zhang L, Arnold WA. Reduction of haloacetic acids by $\mathrm{Fe}^{0}$ : Implications for treatment and fate. Environ. Sci. Technol. 2001;35:2258-2263.

24. Zhang L, Arnold WA, Hozalski RM. Kinetics of haloacetic acid reactions with Fe(0). Environ. Sci. Technol. 2004;38: 6881-6889.

25. Cetó X, Saint C, Chow CWK, Voelcker NH, Prieto-Simón B. Electrochemical fingerprints of brominated trihaloacetic acids (HAA3) mixtures in water. Sen. Act. B 2017;247:70-77.

26. Altamar L, Fernández L, Borras C, Mostany J, Carrero H, Scharifker B. Electroreduction of chloroacetic acids (mono-, di- and tri-) at polyNi(II)-tetrasulfonated phthalocyanine gold modified electrode. Sen. Act. B. 2010;146:103-110.

27. Mao R, Li N, Lan H, et al. Dechlorination of trichloroacetic acid using a noble metal-free Graphene-Cu foam electrode via direct cathodic reduction and atomic $\mathrm{H}^{*}$. Environ. Sci. Technol. 2016;50:3829-3837.

28. Li A, Zhao X, Hou Y, Liu H, Wu L, Qu J. The electrocatalytic dechlorination of chloroacetic acids at electrodeposited $\mathrm{Pd} /$ Fe-modified carbon paper electrode. Appl. Catal. B. 2012;111-112:628-635.

29. Liu Y, Mao R, Tong Y, et al. Reductive dechlorination of trichloroacetic acid (TCAA) by electrochemical process over Pd-In/ $/ \mathrm{Al}_{2} \mathrm{O}_{3}$ catalyst. Electrochim. Acta 2017;232:13-21.

30. Tang S, Wang XM, Yang HW, Xie YF. Haloacetic acid removal by sequential zero-valent iron reduction and biologically active carbon degradation. Chemosphere 2013;90:1563-1567.

31. Zhao X, Li A, Mao R, Liu H, Qu J. Electrochemical removal of haloacetic acids in a three-dimensional electrochemical reactor with Pd-GAC particles as fixed filler and Pd-modified carbon paper as cathode. Water Res. 2014;51:134-143.
32. Li Y, Kemper JM, Datuin G, Akey A, Mitch WA, Luthy RG. Reductive dehalogenation of disinfection byproducts by an activated carbon-based electrode system. Water Res. 2016;98:354-362.

33. Pearson CR, Hozalski RM, Arnold WA. Degradation of chloropicrin in the presence of zero-valent iron. Environ. Toxicol. Chem. 2005;24:3037-3042.

34. Ma J, Yan M, Kuznetsov AM, Masliy AN, Ji G, Korshin GV. Rotating ring-disk electrode and quantum-chemical study of the electrochemical reduction of monoiodoacetic acid and iodoform. Environ. Sci. Technol. 2015;49:13542-13549.

35. Criddle CS, McCarty PL. Electrolytic model system for reductive dehalogenation in aqueous environments. Environ. Sci. Technol. 1991;25:973-978.

36. Sonoyama N, Sakata T. Electrochemical continuous decomposition of chloroform and other volatile chlorinated hydrocarbons in water using a column type metal impregnated carbon fiber electrode. Environ. Sci. Technol. 1999;33: 3438-3442.

37. Polo AMS, López-Peñalver JJ, Sánchez-Polo M, Rivera-Utrilla J, Velo-Gala I, Salazar-Rábago JJ. Oxidation of diatrizoate in aqueous phase by advanced oxidation processes based on solar radiation. J. Photochem. Photobiol. A 2016;319:87-95.

38. Putschew A, Schittko S, Jekel M. Quantification of triiodinated benzene derivatives and X-ray contrast media in water samples by liquid chromatography-electrospray tandem mass spectrometry. J. Chromatogr. A 2001;930:127-134.

39. Ternes TA, Hirsch R. Occurrence and behavior of X-ray contrast media in sewage facilities and the aquatic environment. Environ. Sci. Technol. 2000;34:2741-2748.

40. Stieber M, Putschew A, Jekel M. Treatment of pharmaceuticals and diagnostic agents using zero-valent iron - Kinetic studies and assessment of transformation products assay. Environ. Sci. Technol. 2011;45:4944-4950.

41. Dong H, Qiang Z, Lian J, Li J, Yu J, Qu J. Deiodination of iopamidol by zero valent iron (ZVI) enhances formation of iodinated disinfection by-products during chloramination. Water Res. 2018;129:319-326.

42. Hu J, Dong H, Qu J, Qiang Z. Enhanced degradation of iopamidol by peroxymonosulfate catalyzed by two pipe corrosion products (CuO and $\delta-\mathrm{MnO}_{2}$ ). Water Res. 2017;112:1-8.

43. Mu Y, Radjenovic J, Shen J, Rozendal RA, Rabaey K, Keller J. Dehalogenation of iodinated X-ray contrast media in a bioelectrochemical system. Environ. Sci. Technol. 2011;45: $782-788$

44. Lütke Eversloh C, Schulz M, Wagner M, Ternes TA. Electrochemical oxidation of tramadol in low-salinity reverse osmosis concentrates using boron-doped diamond anodes. Water Res. 2015;72:293-304.

45. Del Moro G, Pastore C, Di Iaconi C, Mascolo G. Iodinated contrast media electro-degradation: Process performance and degradation pathways. Sci. Total Environ. 2015;506-507:631-643.

46. Yan M, Chen Z, Li N, Zhou Y, Zhang C, Korshin G. Electrochemical reductive dehalogenation of iodine-containing contrast agent pharmaceuticals: Examination of reactions of diatrizoate and iopamidol using the method of rotating ring-disc electrode (RRDE). Water Res. 2018;136:104-111. 